\title{
Molecular investigation of an outbreak associated with total parenteral nutrition contaminated with NDM-producing Leclercia adecarboxylata
}

Elvira Garza-González ${ }^{1 \dagger}$, Paola Bocanegra-lbarias ${ }^{1 \dagger}$, Eduardo Rodríguez-Noriega², Esteban González-Díaz², Jesús Silva-Sanchez ${ }^{3}$, Ulises Garza-Ramos ${ }^{3}$, Iván Fernando Contreras-Coronado-Tovar ${ }^{4}$, José Ecil Santos-Hernández ${ }^{4}$, David Gutiérrez-Bañuelos ${ }^{4}$, Juan Pablo Mena-Ramirez ${ }^{5,6}$, Saúl Ramírez-De-los-Santos ${ }^{6}$, Adrián Camacho-Ortiz and Rayo Morfín-Otero $2^{*}$ (D)

\begin{abstract}
Background: This study aimed to determine the epidemiological, microbiological, and molecular characteristics of an outbreak of carbapenem-resistant Leclercia adecarboxylata in three hospitals associated with the unintended use of contaminated total parental nutrition (TPN).

Methods: For 10 days, 25 patients who received intravenous TPN from the same batch of a formula developed sepsis and had blood cultures positive for L. adecarboxylata. Antimicrobial susceptibility and carbapenemase production were performed in 31 isolates, including one from an unopened bottle of TPN. Carbapenemaseencoding genes, extended-spectrum $\beta$-lactamase-encoding genes were screened by PCR, and plasmid profiles were determined. Horizontal transfer of carbapenem resistance was performed by solid mating. Clonal diversity was performed by pulsed-field gel electrophoresis. The resistome was explored by whole-genome sequencing on two selected strains, and comparative genomics was performed using Roary.

Results: All 31 isolates were resistant to aztreonam, cephalosporins, carbapenems, trimethoprim/sulfamethoxazole, and susceptible to gentamicin, tetracycline, and colistin. Lower susceptibility to levofloxacin (51.6\%) and ciprofloxacin (22.6\%) was observed. All the isolates were carbapenemase producers and positive for bla $a_{\text {NDM-1, }}$ bla $a_{\text {TEM-1B }}$, and bla $a_{\mathrm{SHV}-12}$ genes. One main lineage was detected (clone A, 83.9\%; A1, 12.9\%; A2, 3.2\%). The bla $a_{\mathrm{NDM}-1}$ gene is embedded in a Tn125-like element. Genome analysis showed genes encoding resistance for aminoglycosides, quinolones, trimethoprim, colistin, phenicols, and sulphonamides and the presence of IncFII (Yp), IncHI2, and IncHI2A incompatibility groups. (Continued on next page)
\end{abstract}

\footnotetext{
* Correspondence: rayomorfin@gmail.com

${ }^{\dagger}$ Elvira Garza-González and Paola Bocanegra-Ibarias contributed equally to this work.

${ }^{2}$ Hospital Civil de Guadalajara Fray Antonio Alcalde, Instituto de Patología Infecciosa y Experimental, Centro Universitario de Ciencias de la Salud, Universidad de Guadalajara, Guadalajara, Jalisco, Mexico

Full list of author information is available at the end of the article
} changes were made. The images or other third party material in this article are included in the article's Creative Commons licence, unless indicated otherwise in a credit line to the material. If material is not included in the article's Creative Commons licence and your intended use is not permitted by statutory regulation or exceeds the permitted use, you will need to obtain permission directly from the copyright holder. To view a copy of this licence, visit http://creativecommons.org/licenses/by/4.0/ The Creative Commons Public Domain Dedication waiver (http://creativecommons.org/publicdomain/zero/1.0/) applies to the data made available in this article, unless otherwise stated in a credit line to the data. 
(Continued from previous page)

Comparative genomics showed a major phylogenetic relationship among L. adecarboxylata 11 and USDA-ARSUSMARC-60222 genomes, followed by our two selected strains.

Conclusion: We present epidemiological, microbiological, and molecular evidence of an outbreak of carbapenemresistant $L$. adecarboxylata in three hospitals in western Mexico associated with the use of contaminated TPN.

Keywords: NDM-carrying Leclercia adecarboxylata, Outbreak of L. adecarboxylata, Carbapenem-resistant L. adecarboxylata, Contaminated total parenteral nutrition, Bloodstream infections

\section{Background}

Total parenteral nutrition (TPN) is a nutritional supplement for patients unable to receive oral or enteral nutrition. TPN indications mainly include the presence of chronic intestinal obstruction, bowel pseudoobstruction with food intolerance in infants with an immature gastrointestinal system or a congenital gastrointestinal malformation, among others [1] Because manipulation of these supplements may enhance the risk for microbial contamination, absolute compliance to good manufacturing practices is required [2-4]. When these practices are not strictly followed, TPNrelated outbreaks may occur, commonly leading to sepsis with high mortality [5].

Leclercia adecarboxylata is a gram-negative rod with a phenotypic resemblance to Escherichia coli, and Lecler first described it in 1962 as Escherichia adecarboxylata. In 1986 based on DNA hybridization studies, this species was reassigned as part of the Enterobacterales order, Enterobacteriaceae family, and Leclercia genus [6].. This bacterial species has global distribution in a variety of foods, water, and animals and exists as a commensal organism in the gut [7], and has been associated with bacteremia and wound infections and peritonitis, pneumonia, and other infections [8].

Although $L$. adecarboxylata is usually susceptible to antimicrobials, isolates have been reported with resistance to cephalosporins due to extended-spectrum $\beta$ lactamase (ESBL) production [9]. Additionally, $L$. adecarboxylata carbapenem-resistant isolates harboring the $b l a_{\mathrm{NDM}}$ gene have been reported in China and Spain $[10,11]$. Also, a VIM-1 metallo- $\beta$-lactamase has been reported in an isolate of $L$. adecarboxylata from a nonclinical sample [12].

This study aimed to determine the epidemiological, microbiological, and molecular characteristics of an outbreak of carbapenem-resistant $L$. adecarboxylata in three hospitals in western Mexico associated with the unintended use of contaminated TPN.

\section{Methods}

\section{Hospital settings}

This report includes the cases reported by three hospitals in the state of Jalisco: Hospital Civil de Guadalajara
Fray Antonio Alcalde (HC-FAA), Hospital de Pediatría del Centro Médico Nacional de Occidente (HP-CMNO), and Hospital General de Zona 21 (HGZ-21).

The HCG is a 1000-bed tertiary care teaching hospital with a daily occupancy rate of $95 \%$. The HPCMNO is a 205-bed tertiary care teaching hospital with a daily occupancy rate of $93 \%$. Both hospitals serve adult and pediatric populations from the Guadalajara metropolitan area (approximately 4.0 million people) and surroundings states. The HGZ is a 73-bed secondary care hospital with a daily occupancy rate of $97 \%$. This hospital attends most of the Tepatitlán (a city $73 \mathrm{Km}$ from Guadalajara) population with social security (170,701 people). All three hospitals treat only acute conditions.

\section{Epidemiological investigation}

An outbreak started on May 17, when the bacteriology laboratory reported eight blood cultures positive for $L$. adecarboxylata. For 10 days, 25 patients who received intravenous TPN fluids developed sepsis (fever, chills, worsening clinical condition, and leukocytosis) and had blood cultures positive for $L$. adecarboxylata.

The isolation of the same unusual species from patients at several localities indicated that an outbreak was occurring, and an investigation was launched. The finding led the Ministry of Health (Secretaria de Salud) to trigger a nationwide alert, leading to the immediate withdrawal of the TPN formula by the manufacturer.

The implicated vehicles of infection were one batch of TPN formula, supplied in $500-\mathrm{mL}$ bottles. The outbreak was over by May 27, 2019, when the last case presented, and no new cases appeared after.

During the outbreak, a total of $30 \mathrm{~L}$. adecarboxylata isolates were recovered from 25 patients. Additionally, an extensive epidemiological investigation was conducted, tracing the batch number of TPN formula used in all patients, and L. adecarboxylata was isolated in one sealed, unopened bottle of TPN belonging to the same batch administered to the patients. Furthermore, different equipment parts such as bags, connectors, ventilators, and others were submitted to standard microbiological sterility tests, and no microorganisms were recovered. All isolates collected were sent to a reference laboratory for further analysis. 
Identification and antimicrobial susceptibility testing

Identification of isolates was confirmed by matrix-assisted laser desorption ionization time-of-flight mass spectrometry using the Bruker Biotyper system (Bruker Daltonics, Germany) as described previously [13]. Fresh colonies from each strain were spotted onto MALDI-TOF target plates; the spots were covered with $1 \mu \mathrm{l}$ of $70 \%$ formic acid and air-dried. Each spot was overlaid with $1 \mu \mathrm{l}$ of a matrix solution $\alpha$-cyano-4-hydroxycinnamic acid $10 \mathrm{mg} / \mathrm{mL}$ (Sigma Aldrich, Missouri, United States). Samples in spots were analyzed by the Bruker Microflex LT system [14]. The antimicrobial susceptibility was performed by the broth microdilution method using the Clinical \& Laboratory Standards Institute (CLSI) guidelines [15]. Carbapenemase production was detected using the CarbaNP test, according to the CLSI [15].

\section{Molecular methods}

Carbapenemase-encoding genes ( $b l a_{\mathrm{KPC}-\mathrm{type}}, b l a_{\mathrm{VIM}-\mathrm{type}}$, $\left.b l a_{\text {IMP-type }}, b l a_{\text {NDM-type }}, b l a_{\text {OXA-48-type }}\right)$ and ESBL-encoding genes ( $\left.b l a_{\mathrm{TEM}-\mathrm{type}}, b l a_{\mathrm{SHV}-\mathrm{type}}, \quad b l a_{\mathrm{CTX}{ }^{-t y p e}}\right)$ were screened by PCR [16]. Horizontal transfer of carbapenem resistance was performed by solid mating using Escherichia coli J53-2 as the recipient strain [17, 18]. Transconjugants were selected on Luria-Bertani (LB) agar supplemented with rifampicin $(100 \mu \mathrm{g} / \mathrm{mL})$ plus cefoxitin $(30 \mu \mathrm{g} / \mathrm{mL})$. When the conjugation was unsuccessful, the assay was performed in LB agar supplemented with rifampicin $(100 \mu \mathrm{g} / \mathrm{mL})$ plus ceftazidime $(30 \mu \mathrm{g} / \mathrm{mL})$. The plasmid profile was obtained from the $L$. adecarboxylata isolates and transconjugants, according to the method described by Kieser [19].

Clonal diversity was performed using pulsed-field gel electrophoresis following the protocol and conditions previously described using the enzyme SpeI (Takara Bio Inc., Shiga Japón) [20] and interpretation from Tenover et al. criteria [21].

\section{Whole-genome sequencing}

Whole-genome sequencing (WGS) was performed in two selected isolates. DNA was extracted using the DNeasy kit (Qiagen, Germany) according to manufacturer instructions. Sequencing was performed using the Illumina (NextSeq 500) platform. Quality-based trimming was performed with the Trim Galore software, and de novo assembly was completed with SPAdes v3.12.0. The draft genomes were annotated using the NCBI Prokaryotic Genome Annotation Pipeline. The genetic context of the $b l a_{\mathrm{NDM}-1}$ gene was determined by in silico analysis.

\section{Comparative genomics}

The comparative genomics $L$. adecarboxylata genomes included our 16,342 and 16,400 strains, and the deposited USDA-ARS-USMARC-60222, R25, and I1 genomes obtained from a bull, a rabbit, and a human, respectively.

Comparative genomics was performed with the suite Roary using default parameters [22]. The phylogenetic tree's construction based on Roary's core genes alignment was constructed with RAxML v.8 under the GTRG AMMA model [23]. The phylogeny, the pan-genome presence, absence information, and associated metadata were visualized in the Phandango viewer [24]. The ResFinder 3.2 and PlasmidFinder 2.1 tools (http://www. genomicepidemiology.org) were used to identify acquired genes, chromosomal mutations for antibiotic resistance (resistome), and replicon typing of plasmids. In addition, the class 1 integrons were identified using the WGS data on the Integron database (INTEGRAL) (http://integrall.bio.ua.pt/?).

\section{Results}

\section{Affected population and outcome}

All patients, the majority of whom were children (mean age, 78 months or 6.4 years), had been administered TPN via the central line. One patient died (Table 1, supplementary Table 1). The population affected include 23 children and two adults from one of the hospitals. The mean length of stay after a positive culture was 31.2 days, range 3 to 135 days. Before a positive culture, an initial antibiotic more frequently used includes carbapenems, cephalosporins, and aminoglycosides. The termination of TPN and the use of tigecycline was effective in $24 / 25$ patients.

\section{Microbiology}

All $31 \mathrm{~L}$. adecarboxylata isolates were resistant to aztreonam, cefepime, cefotaxime, cefoxitin, ceftazidime, ceftriaxone, ertapenem, imipenem, meropenem, and trimethoprim/sulfamethoxazole. All the strains were susceptible to gentamicin, tetracycline, colistin, lower susceptibility to levofloxacin (51.6\%), and ciprofloxacin (22.6\%) was observed.

\section{Molecular assays}

All 31 isolates [clinical $(n=30)$ and TPN formula $(n=$ 1)] were carbapenemase producers and were positive for $b l a_{\mathrm{NDM}-1}, b l a_{\mathrm{TEM}-\mathrm{type}}$, and $b l a_{\mathrm{SHV}-\mathrm{type}}$ genes, and negative for the other $\beta$-lactamases genes tested. Clinical and TPN isolates harbored three plasmids with approximate sizes of 68-, 124-, and 150-kb. The mating experiments were analyzed and were successful in all isolates; the $b l a_{\mathrm{NDM}-1}$ gene was transferred onto a conjugative plasmid with an approximate 124-kb size.

The genetic relationships of 31 strains isolated indicated one main lineage highly related to the outbreak with three restriction patterns detected: A, (83.9\%, 26/ 
Table 1 Demographic and clinical characteristics of patients affected by the contaminated TPN outbreak in western Mexico

\begin{tabular}{|c|c|c|c|c|}
\hline \multirow[t]{2}{*}{ Characteristic } & \multicolumn{4}{|c|}{ No. $\left(\%\right.$ of patients) ${ }^{a}$} \\
\hline & Total & HC-FAA & HP-CMNO & HGZ-21 \\
\hline No. of patients & 25 & 11 & 1 & 13 \\
\hline Mean age, months (range) & $78(1-996)$ & $152(1-996)$ & 4 & $21(2-168)$ \\
\hline Male & $16(64)$ & $5(45.5)$ & $1(100)$ & $10(76.9)$ \\
\hline \multicolumn{5}{|l|}{ Hospital ward } \\
\hline Neonatal ICU & $19(76)$ & $8(72.7)$ & $1(100)$ & $10(76.9)$ \\
\hline Pediatric surgery & $4(16)$ & $1(9.1)$ & $0(0)$ & $3(23.1)$ \\
\hline General surgery & $1(4)$ & $1(9.1)$ & $0(0)$ & $0(0)$ \\
\hline Internal medicine & $1(4)$ & $1(9.1)$ & $0(0)$ & $0(0)$ \\
\hline Previous hospitalization & $8(32)$ & $2(18.2)$ & $0(0)$ & $6(46.1)$ \\
\hline Mean LOS (days) & $53.8(15-252)$ & $49.1(15-135)$ & 32 & $59.4(30-252)$ \\
\hline Mean LOS to prior positive culture (days) & $22.5(1-249)$ & $10.4(1-22)$ & 14 & $33.5(1-249)$ \\
\hline Mean LOS after positive culture (days) & $31.2(3-135)$ & $39.6(5-135)$ & 18 & $25.1(3-31)$ \\
\hline UCl stay & $20(80)$ & $7(63.6)$ & $1(100)$ & $12(92.3)$ \\
\hline Previous surgery & $10(40)$ & $1(9.1)$ & $0(0)$ & $9(69.2)$ \\
\hline \multicolumn{5}{|c|}{ Number of antibiotics used before positive culture } \\
\hline $1-3$ & $15(60)$ & $6(54.5)$ & $1(100)$ & $8(61.5)$ \\
\hline 4 or more & $10(40)$ & $5(45.5)$ & $0(0)$ & $5(38.5)$ \\
\hline \multicolumn{5}{|l|}{ Antibiotics used before positive culture, n (\%) } \\
\hline Carbapenems & $18(72)$ & $5(45.5)$ & $0(0)$ & $13(100)$ \\
\hline Cephalosporins & $15(60)$ & $11(100)$ & $1(100)$ & $3(23.1)$ \\
\hline Aminoglycoside & $13(52)$ & $8(72.7)$ & $1(100)$ & $4(30.8)$ \\
\hline Glycopeptide & $7(28)$ & $3(27.3)$ & $0(0)$ & $4(30.8)$ \\
\hline Fluoroquinolones & $6(24)$ & $0(0)$ & $0(0)$ & $6(46.1)$ \\
\hline Penicillin & $5(20)$ & $4(36.4)$ & $0(0)$ & $1(7.7)$ \\
\hline Colistin & $4(16)$ & $1(9.1)$ & $0(0)$ & $3(23.1)$ \\
\hline Metronidazole & $3(12)$ & $2(18.2)$ & $0(0)$ & $1(7.7)$ \\
\hline Other & $7(28)$ & $1(9.1)$ & $0(0)$ & $6(46.1)$ \\
\hline Mortality & $1(4)$ & $1(9.1)$ & $0(0)$ & $0(0)$ \\
\hline
\end{tabular}

Abbreviations: HC-FAA Hospital Civil Fray Antonio Alcalde, HP-CMNO Hospital de Pediatría del Centro Médico Nacional de Occidente, HGZ-21 Hospital General de Zona 21, ICU intensive care unit, LOS length of stay, TPN total parenteral nutrition ${ }^{a}$ Mean (range) if otherwise noted

31), A1 $(12.9 \%, 4 / 31)$, and A2 $(2.2 \%, 1 / 31)$, with a percentage of similarity ranging from 90 to $100 \%$.

\section{Resistome identified by whole-genome sequencing}

The WGS was performed in two selected isolates: 16342 (strain A) and 16,400 (strain B), both strains from patients, and generated a total of 2,893,824 (A) and 2,406, 492 (B) pair-end reads with a length of $75 \mathrm{bp}$. In total, 131 (A) and 150 (B) contigs with an N50 of 93,591 bp (A) and $108,425 \mathrm{bp}$ (B) were obtained. The estimated size genome sized were 5,232,567 bp (A) and 5,299,868 bp (B) with $120 \times$ coverage.

The resistome on both strains had genes encoding resistance for aminoglycosides (aac[6']-Ib3, aadA2b, $\operatorname{aph}[3 "]-I b, \quad \operatorname{aph}\left[3^{\prime}\right]-I a$ and $\left.a p h[6]-I d\right), \quad \beta$-lactam $\left(b l a_{\mathrm{NDM}-1}, \quad b l a_{\mathrm{TEM}-1 \mathrm{~B}}, \quad\right.$ and $\left.b l a_{\mathrm{SHV}-12}\right)$, quinolones (aac [6']-Ib-cr), trimethoprim (dfrA19), colistin (mor-9), phenicols (catA2), and sulphonamides (sul1) (Supplementary Table 2). Both strains A as B the genetic context revealed the $\triangle \mathrm{ISAba125-NDM-1-bleMBL-}$

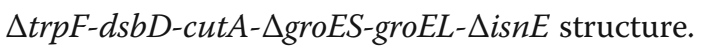

Additionally, the A strain included the qurS2 gene (encoding resistance for nalidixic acid), and the B strain included the qnrB2 gene (encoding resistance for nalidixic acid) and the $d f r A 12$ gene (encoding resistance for trimethoprim). The class 1 integron identified showed aminoglycoside (aadA2 and, aacA4) and trimethoprim (dfrA12) resistance genes. $d f r A 12$ is part of a new gene 
arrangement of the integron In1982 and the $g c u F \Delta 23$, a gene cassette of unknown function (Supplementary Table 3).

The replicon typing of plasmid analysis identified in both genomes had the IncFII (Yp) (99.13\% identity), IncHI2 (100\% identity), and IncHI2A (100\% identity) incompatibility groups (Supplementary Table 4).

\section{Genomic comparison of $L$. adecarboxylata genomes}

A major phylogenetic relationship was observed among I1 and USDA-ARS-USMARC-60222 isolates, followed by 16,342 (strain A) and 16,400 (strain B) (Fig. 1). However, the resistome identified in the genome in silico is different among these isolates. In the $\mathrm{I} 1$ and $\mathrm{A}$, and $\mathrm{B}$ genomes, a large number of resistance genes were identified (Fig. 1). The incompatibility group was the same in $\mathrm{A}, \mathrm{B}$, and R25 and different in the I1 genome (carrying NDM-1) (Fig. 1).

\section{Discussion}

Several reports have described sepsis outbreaks associated with substances administrated to patients involving Staphylococcus saprophyticus, Enterobacter spp., Acinetobacter spp., Pantoea agglomerans, Burkholderia cepacia, and Candida albicans [25-28]. Among these outbreaks, one of the most frequently reported causes is TPN's use, with most of them being related to the colonization of intravenous cannula. Outbreaks of infection are rarely associated with TPN products contaminated before they reached the patient. No previous report has described outbreaks associated with the use of contaminated TPN and $L$. adecarboxylata, thus demonstrating our report's novelty. Additionally, we verified by molecular methods that the isolates collected from patients and the one from the unopened bottle were one main lineage highly related to the outbreak, with a clone $\mathrm{A}$ and two subtypes.

Bloodstream infection incidence in patients receiving TPN has been reported to be as high as 39\% [29]. In our study, most affected patients were children, and most of them were in the neonatal intensive care unit (ICU) ward. It has been described that the administration of TPN through parenteral catheter represents a risk of 4.69 for bloodstream infections in neonates [30, 31]. In our study, unfortunately, one patient died of sepsis.

A review of 74 cases of infections associated with $L$. adecarboxylata (including bacteremia) showed high susceptibility to antibiotics, and treatment was efficient in most cases [8]. Two pediatric cases of cellulitis and urinary tract infection associated with $L$. adecarboxylata were also successfully treated with antibiotics. These reports highlight the importance of this outbreak because clinical isolates related to this outbreak were highly resistant to antibiotics and the need to become more aware of its threat in pediatric and adult populations.

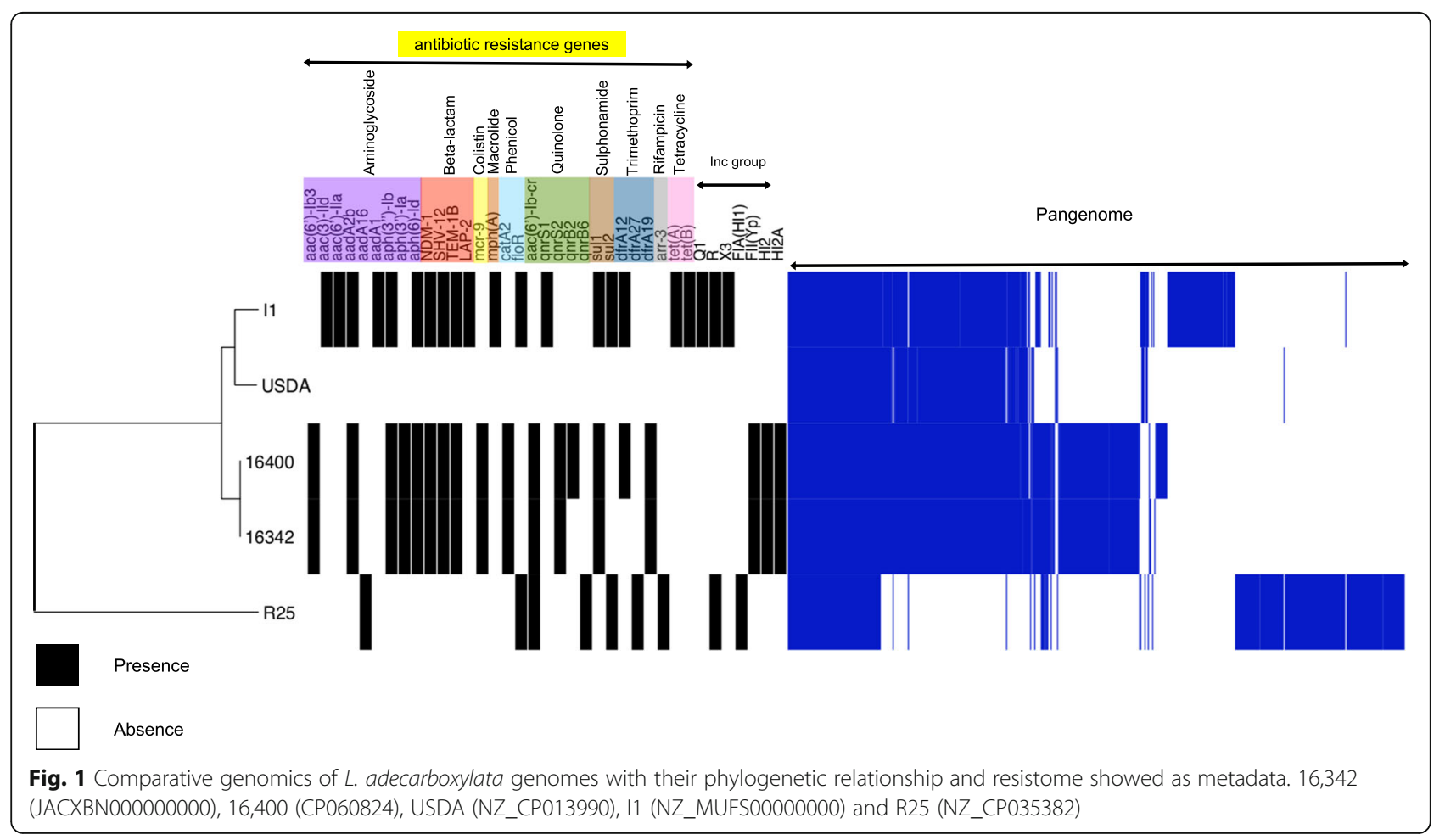


Most of the patients were receiving carbapenems and cephalosporins before the outbreak, and according to microbiological results, the $L$. adecarboxylata isolates were resistant to these drugs, which may have helped to increase the severity of the outbreak.

The genetic context of the $b l a_{\mathrm{NDM}-1}$ identified in this study was identical to the Tn125-like described on a pP10164-DNM plasmid from $L$. adecarboxylata P10164 isolates described previously in China [10].

Infections caused by carbapenemase-producing bacteria are difficult to treat, with the selection of initial or definitive appropriate therapy being problematic [32, 33]. The driving force for the development of resistant bacteria is the inappropriate use of antibiotics, a factor that can be forestalled with the use of sustained antimicrobial stewardship [34, 35], and the early identification and containment are crucial to help efforts [34]. A recent report looking at antimicrobial resistance in Mexico derived from 47 centers in 20 states, during 6 months in a total sample of 22,943 strains analyzed, found carbapenem resistance in $12.5 \%$ of Klebsiella sp. and Enterobacter sp., and 40\% in Pseudomonas aeruginosa [36].

The class 1 integrons identified in L. adecarboxylata genomes contain genes related to aminoglycoside resistance, resulting in a new gene array in the In1982 class 1 integron. Integrons' presence has been broadly described among Enterobacterales isolates, which use site-specific recombination to move resistance genes between defined sites $[37,38]$. Also, mobile elements are involved in the spread of integrons [39].

Interestingly, both strains had genes encoding aminoglycoside resistance but were susceptible to gentamicin and had the $m c r-9$ gene and were susceptible to colistin. Previous studies have identified $m c r$-4-bearing plasmids in L. adecarboxylata from human gut microbiota [10, 40], but no study had reported the association of the mor genotype and the colistin resistance in this bacterial species. These findings highlight the need for epidemiological-molecular studies because strains may render as drug-resistant organisms by the selective pressure exerted by antibiotics.

Although 16,342 (strain A) and 16,400 (strain B) genomes were identified as one clone using PFGE, they showed slight differences in their pan-genome at the genomic level. Thus, the $q n r A$ and $d f r A 12$ genes were absent in the A genome.

The A and B genomes showed an MDR resistome related to isolates from clinical settings, as was the case for the human collected genome I1.

In a recent study that included $34 \mathrm{~L}$. adecarboxylata isolates collected from 2005 to 2017, 18 were considered clinically significant pathogens, and 16 were considered contaminants [41]; showing that this organism may be easily isolated as contaminants. In pour study, the isolates were collected from blood, so there were clinically significant.

Our study has some limitations because only two strains were sequenced, and surveillance was not performed in all hospitals that received the same batch of bottles.

\section{Conclusions}

Outbreaks are most likely to occur when hospital staff does not adhere to basic hygiene measures. In our study, the contamination observed was detected in unopened TPN bottles, and the strain from the new bottle was identified by molecular methods to match the strain isolated from patients. Our results reinforce the importance of an in-depth epidemiological, microbiological, and molecular characterization before attributing an outbreak to a hospital's nonadherence to hygiene measures. Continuous adherence to hygiene protocols and surveillance of unopened bottles may help to reduce outbreaks.

\section{Supplementary Information}

The online version contains supplementary material available at https:/doi. org/10.1186/s12879-021-05923-0.

Additional file 1: S1 Table. Demographic characteristic of patients. Additional file 2: S2 Table. Antibiotics resistance family genes identified in the genomes of L. adecarboxylata included in the analysis. Additional file 3: S3 Table. Class 1 integron identified in the $L$. adecarboxylata 16,342 and 16,400 genomes.

Additional file 4: S4 Table. Incompatibility groups identified in the genomes of $L$. adecarboxylata included in the analysis.

\section{Abbreviations}

TPN: Total Parenteral Nutrition; HC-FAA: Hospital Civil Fray Antonio Alcalde; HP-CMNO: Hospital de Pediatria del Centro Medico Nacional de Occidente: HGZ-21: Hospital General de Zona 21; ICU: Intensive Care Unit; LOS: Length of stay; WGS: Whole-genome sequencing

\section{Acknowledgments}

We acknowledge Alejandro Sánchez for technical support.

\section{Authors' contributions}

Conceived and designed the study EGG, PBI, RMO, ERN, JSS, UGR, Participated in sample collection, epidemiological investigation: EGG, PBI, RMO, EGD, ICC, JSH, DGB, JMR, SRS, ACO. Participated in experiment designing and performed the experiments: EGG, PBI, JSS, JSS, UGR. Manuscript preparation and manuscript correction: EGG, PBI, RMO, JSS, UGR, ERN, ACO. All authors read and approved the final manuscript. Participated in experiment designing and manuscript correction.

Funding

This study was not funded.

\section{Availability of data and materials}

The datasets generated and/or analyzed during the current study are available from GenBank under the accession numbers: JACXBN000000000, CP060824, CP013990, MUFS00000000, NZ_CP035382.

\section{Ethics approval}

The local ethics committee (Comité de Ética en Investigación del Antiguo Hospital Civil de Guadalajara Fray Antonio Alcalde, Jalisco, Mexico) approved 
this study (reference number 062/19). This committee waived informed consent because no intervention was involved, and no patient identifying information was included.

\section{Consent for publication}

Not applicable.

\section{Competing interests}

The authors declare that they have no competing interests relevant to this article.

\section{Author details}

${ }^{1}$ Hospital Universitario Dr. José Eleuterio González, Universidad Autónoma de Nuevo León, Monterrey, Nuevo León, Mexico. ${ }^{2}$ Hospital Civil de Guadalajara Fray Antonio Alcalde, Instituto de Patología Infecciosa y Experimental, Centro Universitario de Ciencias de la Salud, Universidad de Guadalajara, Guadalajara, Jalisco, Mexico. ${ }^{3}$ Centro de Investigación Sobre Enfermedades Infecciosas, Instituto Nacional de Salud Pública, Cuernavaca, Morelos, Mexico. ${ }^{4}$ Hospital de Pediatría de Centro Médico Nacional de Occidente, Guadalajara, Jalisco, Mexico. ${ }^{5}$ Hospital General de Zona No.21 IMSS, Centro Universitario de los Altos (CUALTOS), Universidad de Guadalajara, Tepatitlán de Morelos, Jalisco, Mexico. ${ }^{6}$ Instituto de Investigación en Biociencias, Centro Universitario de los Altos, Universidad de Guadalajara, Tepatitlán de Morelos, Jalisco, Mexico.

\section{Received: 30 October 2020 Accepted: 19 February 2021}

\section{Published online: 28 February 2021}

\section{References}

1. Chowdary KV, Reddy PN. Parenteral nutrition: revisited. Indian J Anaesth. 2010;54(2):95-103.

2. Driscoll DF. Compounding TPN admixtures: then and now. JPEN J Parenter Enteral Nutr. 2003;27(6):433-8 quiz 439.

3. Vonberg RP, Gastmeier P. Hospital-acquired infections related to contaminated substances. J Hosp Infect. 2007;65(1):15-23.

4. Gupta N, Hocevar SN, Moulton-Meissner HA, Stevens KM, McIntyre MG, Jensen B, Kuhar DT, Noble-Wang JA, Schnatz RG, Becker SC, et al. Outbreak of Serratia marcescens bloodstream infections in patients receiving parenteral nutrition prepared by a compounding pharmacy. Clin Infect Dis. 2014;59(1):1-8

5. Pillonetto M, Arend L, Gomes SMT, Oliveira MAA, Timm LN, Martins AF, Barth AL, Mazzetti A, Hersemann L, Smits THM, et al. Molecular investigation of isolates from a multistate polymicrobial outbreak associated with contaminated total parenteral nutrition in Brazil. BMC Infect Dis. 2018;18(1): 397.

6. Adeolu M, Alnajar S, Naushad SS, Gupta R. Genome-based phylogeny and taxonomy of the 'Enterobacteriales': proposal for Enterobacterales ord. nov. divided into the families Enterobacteriaceae, Erwiniaceae fam. nov. Pectobacteriaceae fam. nov., Yersiniaceae fam. nov., Hafniaceae fam. nov., Morganellaceae fam. nov., and Budviciaceae fam. nov. Int J Syst Evol Microbiol. 2016;66(12):5575-99.

7. Garcia-Fulqueiras $V$, Seija V, Aguerrebere P, Cordeiro NF, Vignoli R. First report of a clinical isolate of Leclercia adecarboxylata harbouring multiple resistance genes in Uruguay and review of the literature. J Glob Antimicrob Resist. 2014:2(2):77-81.

8. Spiegelhauer MR, Andersen PF, Frandsen TH, Nordestgaard RLM, Andersen LP. Leclercia adecarboxylata: a case report and literature review of 74 cases demonstrating its pathogenicity in immunocompromised patients. Infect Dis (Lond). 2019:51(3):179-88.

9. Alosaimi RS, Muhmmed Kaaki M. Catheter-related ESBL-producing. Case Rep Infect Dis. 2020;2020:7403152

10. Sun F, Yin Z, Feng J, Qiu Y, Zhang D, Luo W, Yang H, Yang W, Wang J, Chen W, et al. Production of plasmid-encoding NDM-1 in clinical Raoultella ornithinolytica and Leclercia adecarboxylata from China. Front Microbiol. 2015;6:458.

11. Riazzo C, Lopez-Cerero L, Rojo-Martin MD, Hoyos-Mallecot Y, FernandezCuenca F, Martin-Ruiz JL, Pascual-Hernandez A, Naas T, Navarro-Mari JM. First report of NDM-1-producing clinical isolate of Leclercia adecarboxylata in Spain. Diagn Microbiol Infect Dis. 2017;88(3):268-70.

12. Papagiannitsis CC, Studentová V, Hrabák J, Kubele J, Jindrák V, Zemlicková H. Isolation from a nonclinical sample of Leclercia adecarboxylata producing a VIM-1 metallo- $\beta$-lactamase. Antimicrob Agents Chemother. 2013;57(6): 2896-7.

13. Levesque S, Dufresne PJ, Soualhine H, Domingo MC, Bekal S, Lefebvre B, Tremblay C. A side by side comparison of Bruker Biotyper and VITEK MS: utility of MALDI-TOF MS Technology for Microorganism Identification in a public health reference laboratory. Plos One. 2015;10(12):e0144878.

14. Schubert S, Kostrzewa M. MALDI-TOF MS in the microbiology laboratory: current trends. Curr Issues Mol Biol. 2017;23:17-20.

15. Clinical and Laboratory Standards Institute: Performance Standards for Antimicrobial Susceptibility Testing; 30th Edition Informational Supplement M100-S20 2020(1).

16. Bocanegra-Ibarias P, Garza-Gonzalez E, Padilla-Orozco M, Mendoza-Olazaran S, Perez-Alba E, Flores-Trevino S, Garza-Ramos U, Silva-Sanchez J, CamachoOrtiz A. The successful containment of a hospital outbreak caused by NDM1-producing Klebsiella pneumoniae ST307 using active surveillance. Plos One. 2019;14(2):e0209609.

17. Hamprecht A, Poirel L, Gottig S, Seifert H, Kaase M, Nordmann P. Detection of the carbapenemase GIM-1 in Enterobacter cloacae in Germany. J Antimicrob Chemother. 2013;68(3):558-61.

18. Miller $\mathrm{JH}$. Experiments in molecular genetics. Cold Spring Harbor: Cold Spring Harbor Laboratory; 1972.

19. Kieser T. Factors affecting the isolation of CCC DNA from Streptomyces lividans and Escherichia coli. Plasmid. 1984;12(1):19-36.

20. Kaufmann ME. Pulsed-field gel electrophoresis. Methods Mol Med. 1998;15: 33-50.

21. Tenover FC, Arbeit RD, Goering RV, Mickelsen PA, Murray BE, Persing DH, Swaminathan B. Interpreting chromosomal DNA restriction patterns produced by pulsed-field gel electrophoresis: criteria for bacterial strain typing. J Clin Microbiol. 1995:33(9):2233-9.

22. Page AJ, Cummins CA, Hunt M, Wong VK, Reuter S, Holden MT, Fookes M, Falush D, Keane JA, Parkhill J. Roary: rapid large-scale prokaryote pan genome analysis. Bioinformatics. 2015;31(22):3691-3.

23. Stamatakis A. RAxML version 8: a tool for phylogenetic analysis and postanalysis of large phylogenies. Bioinformatics. 2014;30(9):1312-3.

24. Hadfield J, Croucher NJ, Goater RJ, Abudahab K, Aanensen DM, Harris SR. Phandango: an interactive viewer for bacterial population genomics. Bioinformatics. 2018;34(2):292-3.

25. Llop JM, Mangues I, Perez JL, Lopez P, Tubau M. Staphylococcus saprophyticus sepsis related to total parenteral nutrition admixtures contamination. JPEN J Parenter Enteral Nutr. 1993;17(6):575-7.

26. McGrath EJ, Chopra T, Abdel-Haq N, Preney K, Koo W, Asmar BI, Kaye KS. An outbreak of carbapenem-resistant Acinetobacter baumannii infection in a neonatal intensive care unit: investigation and control. Infect Control Hosp Epidemiol. 2011;32(1):34-41.

27. Guducuoglu H, Gultepe B, Otlu B, Bektas A, Yildirim O, Tuncer O, Berktas M. Candida albicans outbreak associated with total parenteral nutrition in the neonatal unit. Indian J Med Microbiol. 2016;34(2):202-7.

28. Campos LC, Lobianco LF, Seki LM, Santos RM, Asensi MD. Outbreak of Enterobacter hormaechei septicaemia in newborns caused by contaminated parenteral nutrition in Brazil. J Hosp Infect. 2007;66(1):95-7.

29. Turpin RS, Canada T, Rosenthal V, Nitzki-George D, Liu FX, Mercaldi CJ, Pontes-Arruda A, Group IS. Bloodstream infections associated with parenteral nutrition preparation methods in the United States: a retrospective, large database analysis. JPEN J Parenter Enteral Nutr. 2012; 36(2):169-76.

30. Távora AC, Castro AB, Militão MA, Girão JE, KeC R, Távora LG. Risk factors for nosocomial infection in a Brazilian neonatal intensive care unit. Braz J Infect Dis. 2008;12(1):75-9.

31. Perlman SE, Saiman L, Larson EL. Risk factors for late-onset health careassociated bloodstream infections in patients in neonatal intensive care units. Am J Infect Control. 2007:35(3):177-82.

32. Logan LK, Weinstein RA. The Epidemiology of Carbapenem-Resistant Enterobacteriaceae: The Impact and Evolution of a Global Menace. J Infect Dis. 2017;215(suppl_1):S28-36.

33. Escandón-Vargas K, Reyes S, Gutiérrez S, Villegas MV. The epidemiology of carbapenemases in Latin America and the Caribbean. Expert Rev Anti-Infect Ther. 2017;15(3):277-97.

34. Goff DA, Kullar R, Goldstein EJC, Gilchrist M, Nathwani D, Cheng AC, Cairns KA, Escandón-Vargas K, Villegas MV, Brink A, et al. A global call from five countries to collaborate in antibiotic stewardship: united we succeed, divided we might fail. Lancet Infect Dis. 2017;17(2):e56-63. 
35. Hegewisch-Taylor J, Dreser-Mansilla A, Romero-Mónico J, Levy-Hara G. Antimicrobial stewardship in hospitals in Latin America and the Caribbean: a scoping review. Rev Panam Salud Publica. 2020;44:e68.

36. Garza-González E, Morfín-Otero R, Mendoza-Olazarán S, Bocanegra-Ibarias $P$, Flores-Treviño S, Rodríguez-Noriega E, Ponce-de-León A, Sanchez-Francia D, Franco-Cendejas R, Arroyo-Escalante S, et al. A snapshot of antimicrobial resistance in Mexico. Results from 47 centers from 20 states during a sixmonth period. Plos One. 2019;14(3):e0209865.

37. Partridge SR, Kwong SM, Firth N, Jensen SO: Mobile Genetic Elements Associated with Antimicrobial Resistance. Clin Microbiol Rev 2018;31(4): E00088-17.

38. Boucher $Y$, Labbate M, Koenig JE, Stokes HW. Integrons: mobilizable platforms that promote genetic diversity in bacteria. Trends Microbiol. 2007; 15(7):301-9.

39. Miriagou V, Douzinas EE, Papagiannitsis CC, Piperaki E, Legakis NJ,

Tzouvelekis LS. Emergence of Serratia liquefaciens and Klebsiella oxytoca with metallo-beta-lactamase-encoding IncW plasmids: further spread of the blaVIM-1-carrying integron in-e541. Int J Antimicrob Agents. 2008;32(6):540-1.

40. Sun Q, Wang H, Shu L, Dong N, Yang F, Zhou H, Chen S, Zhang R. From human gut Flora carries. Front Microbiol. 2019;10:2805.

\section{Publisher's Note}

Springer Nature remains neutral with regard to jurisdictional claims in published maps and institutional affiliations.

Ready to submit your research? Choose BMC and benefit from:

- fast, convenient online submission

- thorough peer review by experienced researchers in your field

- rapid publication on acceptance

- support for research data, including large and complex data types

- gold Open Access which fosters wider collaboration and increased citations

- maximum visibility for your research: over $100 \mathrm{M}$ website views per year

At $\mathrm{BMC}$, research is always in progress.

Learn more biomedcentral.com/submissions 